\title{
UNION DEFENCE FORCES : STATISTICS OF THE WOUNDED AND PRISONERS OF WAR DURING THE SECOND WORLD WAR 1939-1945 Compiled by Captain J.E. Loraine-Grews
}

In 1943, after active service as RQMS of Die Middellandse Regiment in the Western Desert during which he escaped capture at Tobruk and was present at the battle of El Alamein, S/Sgt later Capt J.E. Loraine-Grews was posted to War Records (now Military Information Bureau SADF) in Pretoria where he was to remain until 1966 when he was transferred to the Military Museum at the Castle in Cape Town.

While at War Records he compiled the Union of South Africa Roll of Honour 1939-1945. Capt LoraineGrews also compiled statistics of the wounded and prisoners of war and since these have never been published, may be of value to researchers and would be a monumental task for a researcher to compile afresh. They are published below.

\begin{tabular}{|c|c|c|c|c|c|}
\hline \multirow{2}{*}{ UNIT } & \multicolumn{5}{|c|}{ EDP WOUNDED PERSONNEL AND THEATRE OF WAR } \\
\hline & POW & EAST AFRICA & NORTH AFRICA & ITALY & $\begin{array}{l}\text { MISCEL- } \\
\text { LANEOUS }\end{array}$ \\
\hline $\begin{array}{l}\text { Staff Corps (V) } \\
\text { SA Tank Corps } \\
\text { SAEC } \\
\text { SAMC } \\
\text { TSC } \\
\text { QSC } \\
\text { SAA } \\
\text { SACS } \\
\text { GSC } \\
\text { SA Irish } \\
\text { DEOR } \\
\text { RB } \\
\text { FFB } \\
\text { TS } \\
\text { RNC } \\
\text { NMR } \\
\text { ILH } \\
\text { RLI } \\
\text { RDLI } \\
\text { CTH } \\
\text { RPS } \\
\text { SAP } \\
\text { KAFF R } \\
\text { DMR } \\
\text { UMR } \\
\text { SACMP } \\
\text { Armoured car units } \\
\text { WR/DLR } \\
\text { Seconded personnel } \\
\text { FC/CTH } \\
\text { ILH/KR } \\
\text { PR } \\
\text { PAG } \\
\text { SSB } \\
\text { NMR/SAAF } \\
\text { SAAF } \\
\text { SANF } \\
\text { CC } \\
\text { IMC } \\
\text { NMC }\end{array}$ & $\begin{array}{r}22 \\
595 \\
249 \\
598 \\
438 \\
2326 \\
855 \\
180 \\
203 \\
114 \\
532 \\
28 \\
913 \\
104 \\
53 \\
185 \\
223 \\
736 \\
63 \\
96 \\
1080 \\
532 \\
439 \\
532 \\
145 \\
550 \\
\end{array}$ & $\begin{array}{r}11 \\
9 \\
3 \\
2 \\
2 \\
12 \\
3 \\
1 \\
1 \\
8 \\
45 \\
11 \\
12 \\
38 \\
21 \\
16\end{array}$ & $\begin{array}{r}212 \\
60 \\
56 \\
60 \\
616 \\
99 \\
33 \\
45 \\
64 \\
177 \\
198 \\
342 \\
128 \\
160 \\
155 \\
185 \\
188 \\
145 \\
64 \\
103 \\
42 \\
18 \\
16 \\
14 \\
222\end{array}$ & $\begin{array}{r}12 \\
580 \\
\\
327 \\
432 \\
138 \\
124 \\
167 \\
10\end{array}$ & $\begin{array}{r}16 \\
4 \\
8 \\
1 \\
51 \\
13 \\
3 \\
13 \\
1 \\
6 \\
\\
9 \\
1 \\
1 \\
6 \\
15 \\
4 \\
5 \\
12 \\
6 \\
4 \\
2 \\
15 \\
\\
158\end{array}$ \\
\hline & 17303 & 198 & 3741 & 3319 & 613 \\
\hline
\end{tabular}

